\title{
Influência do tempo de indução nas propriedades de hidroxiapatita porosa obtida por gelcasting de espumas
}

\section{(Influence of the induction time on the properties of porous hydroxyapatite obtained by gelcasting foams)}

\author{
T. M. Volkmer, L. A. dos Santos \\ Departamento de Materiais, Universidade Federal do Rio Grande do Sul, Porto Alegre, RS 91510-970 \\ tiagovolkmer@gmail.com
}

\begin{abstract}
Resumo
Hidroxiapatita é uma cerâmica bioativa que permite o crescimento de tecido ósseo e revascularização da área implantada pela formação de ligação com a fase mineral dos ossos. Este estudo teve como objetivo a obtenção de blocos porosos de hidroxiapatita através do método gelcasting de espuma, sem a utilização de atmosfera controlada para a realização das polimerizações. O processo gelcasting de espumas consiste na incorporação de uma fase gasosa dispersa dentro de uma suspensão cerâmica contendo o pó cerâmico, água, defloculantes, ligantes e agentes de gelificação. O sistema de gelificação consiste no monômero acrilamida e no comonômero N,N' metilenobisacrilamida difuncional (MBAM). Foi estudada a variação das quantidades de iniciador e de acelerador e verificou-se a variação do tempo de indução e seus reflexos nas propriedades da hidroxiapatita porosa. Avaliou-se a porosidade, a macroestrutura, a distribuição de tamanho de poros e a permeabilidade. Verificou-se que é possível controlar a porosidade variandose o tempo de indução e a ausência de atmosfera controlada não impediu a obtenção das peças porosas.

Palavras-chave: gelcasting de espumas, hidroxiapatita, biocerâmica, atmosfera, polimerização.
\end{abstract}

\begin{abstract}
Hydroxyapatite (HA) is a bioactive ceramic that allows bone growth and revascularization of the area of implantation trough the formation of chemical bonding with the mineral phase of the bones. The aim of this work was to obtain porous blocks of HA by the gelcasting foams method without atmosphere control. The gelcasting foams process consists in the incorporation of a dispersed gaseous phase, with the help of a surfactant, inside a ceramic suspension containing the ceramic powder, water and gelation agents. The gelation system is composed by one monomer, acrylamide, and one comonomer, methylenebisacrylamide (MBAM). The effect of the accelerator and initiator amounts on the induction time, porosity, pore size distribution and permeability was evaluated. It was possible to control the porosity through the variation of the induction time by changing the amounts of reactions initiator and accelerator, and the absence of controlled atmosphere did not impede the preparation of HA porous blocks.

Keywords: bioceramics, gelcasting foams, permeability, atmosphere, polimerization.
\end{abstract}

\section{INTRODUÇ̃̃O}

O tecido ósseo tem a capacidade de regeneração com a produção de novo tecido com a mesma alta organização estrutural original. No entanto, esta capacidade regenerativa é limitada pelo tamanho da lesão. Assim, defeitos ósseos extensos, provocados por traumas, infecções, neoplasias e anomalias de desenvolvimento não se regeneram espontaneamente, representando um problema atual na medicina e odontologia. Por possuir similaridade química com a fase mineral dos tecidos ósseos, a hidroxiapatita é um dos materiais mais biocompatíveis conhecidos, favorecendo o crescimento ósseo para os locais em que ela se encontra (osteocondutor), estabelecendo ligações entre a hidroxiapatita e o tecido ósseo (bioativo), permitindo a proliferação de fibroblastos, osteoblastos e outras células ósseas, sendo que as células não distinguem entre a hidroxiapatita e a superfície óssea, o que indica a grande similaridade química superficial. Ela também possui a capacidade de trocar íons com o meio fisiológico levando ao equilíbrio entre implante e osso [1,2]. A superfície da hidroxiapatita permite a interação de ligações do tipo dipolo, fazendo com que moléculas de água e também, proteínas e colágeno sejam adsorvidas na superfície induzindo, assim, a regeneração tecidual [3].

Os poros fornecem local para o tecido ósseo crescer e fixar o implante biologicamente. O tamanho mínimo necessário para o crescimento de células ósseas é de 100-200 $\mu \mathrm{m}$. Poros menores entre 75 e $100 \mu \mathrm{m}$ resultam no crescimento de tecido osteóide não mineralizado. Poros menores ainda, entre 10 e $75 \mu \mathrm{m}$, permitem a penetração apenas de tecido fibroso, o que ajuda na fixação mecânica da peça [4].

$\mathrm{O}$ processo gelcasting de espumas consiste na incorporação de uma fase gasosa dispersa dentro de uma suspensão cerâmica contendo o pó cerâmico, água, defloculantes, ligantes e agentes de geleificação. A 
incorporação pode ser feita através de agitação da suspensão. Após a etapa de formação de espuma o material é geleificado pela polimerização in situ de monômeros orgânicos $[5,6]$ e o controle do tempo de geleificação é primordial para a obtenção de porosidade controlada. Após a formação da espuma, a polimerização deve ser rápida o suficiente para prevenir o colapso desta, que ocorre pela drenagem do líquido pela força capilar e pela gravitacional. Finalmente, a rede polimérica formada deve ser suficientemente forte para suportar a estrutura porosa. Apesar de sua alta porosidade, a espuma geleificada deve resistir à desmoldagem, ao manuseamento, à secagem e usinagem.

Este estudo teve como objetivo a obtenção de blocos porosos de hidroxiapatita através do método gelcasting de espumas, sem o uso de atmosfera controlada e utilizando pó sintetizado apenas com matérias-primas nacionais. Estudouse a variação dos teores de catalisador e de iniciador e sua influencia nas propriedades do material final. Caracterizouse por microscopia eletrônica de varredura a morfologia, tamanho e distribuição de tamanho dos poros, sendo este último pouco reportado na literatura. A porosidade foi medida pelo método geométrico. A permeabilidade dos blocos porosos também foi medida. Difração de raios $\mathrm{X}$ foi utilizada para caracterizar a estrutura cristalina e a presença de outras fases na HA precipitada.

\section{MATERIAIS E MÉTODOS}

A hidroxiapatita utilizada neste trabalho foi obtida pelo método via-úmida a partir de matérias primas nacionais e com adição de defloculante, método inédito [7]. Posteriormente a HA foi calcinada a $1200^{\circ} \mathrm{C}$. Depois de calcinada, foi moída em álcool etílico por $24 \mathrm{~h}$ para diminuição do tamanho de partículas e aumento da área superficial. A suspensão de hidroxiapatita foi feita em uma solução aquosa de monômero (pré-mix) composta pelo monômero orgânico acrilamida $\left(\mathrm{C}_{6} \mathrm{H}_{5} \mathrm{NO}\right)$, Vetec, pelo comonômero formador de ligações cruzadas N,N' metilenobisacrilamida difuncional (MBAM), Sigma-Aldrich Corp., e pelo dispersante poliacrilato de amônia Disperlan L.A (Lambra S/A). O agente surfactante utilizado foi o Lutensol ON 110 (Basf). O iniciador utilizado foi o persulfato de amônia $\left(98 \%+\operatorname{ACS}\left(\mathrm{NH}_{4}\right)_{2} \mathrm{~S}_{2} \mathrm{O}_{8}\right)$. Já o acelerador foi N,N,N',N'-tetrametiletilenodiamina (TEMED). Todas as suspensões foram feitas em água destilada e deionizada. Na Tabela I são mostrados os teores de cada constituinte da Pré-mix.

Tabela I - Percentual e função dos elementos da Pré-mix em relação à quantidade de água destilada e deionizada.

[Table 1 - Amounts and function of pré-mix elements.]

\begin{tabular}{lcc}
\hline Elemento & Quantidade (\%) & Função \\
\hline Acrilamida & 20 & Monômero \\
$\begin{array}{l}\text { N,N' metilenobisacrilamida } \\
\text { difuncional (MBAM) }\end{array}$ & 0,4 & Comonômero \\
\begin{tabular}{l} 
Poliacrilato de Amônia \\
\hline
\end{tabular} & 3 & Dispersante \\
\hline
\end{tabular}

Para verificar a presença de outras fases na hidroxiapatita foram feitas análises de difração de raios $\mathrm{X}$ (difratômetro Phillips X'Pert MPD, tubo $\mathrm{CuK} \square$ ) e espectroscopia de infravermelho (FTIR). Para a formação da espuma foi utilizado $1 \%$ em peso do agente surfactante Lutensol ON 110 (C10-Ox0 álcool+11 EO) [8], um surfactante não iônico que é um álcool etoxilato, utilizando-se como meio a solução aquosa de monômero pré-mix. E as suspensões preparadas possuíam 62\% de hidroxiapatita [9]. Posteriormente, a suspensão foi agitada a $600 \mathrm{rpm}$ [10] por 4 min para a formação de uma espuma cerâmica.

A formação do gel se deu pala adição de diferentes quantidades do iniciador, persulfato de amônia (APS), e do acelerador, N,N,N',N'-tetrametiletilenodiamina (TEMED). Essa adição posterior de TEMED é feita para controlar o tempo de polimerização, fator extremamente importante para a reprodutibilidade dos resultados. As reações de polimerização foram realizadas sem controle de atmosfera, assim como, foram feitas no mesmo dia para evitar diferenças de temperatura e de umidade que pudessem interferir nos resultados. O processo gelcasting de espumas normalmente utiliza atmosfera controlada, uma vez que o oxigênio interfere na reação de polimerização [11]. A não utilização de atmosfera visou avaliar a real necessidade deste controle no processo de obtenção de biocerâmicas porosas e potencialmente reduzir o seu custo. O controle do tempo de indução foi feito com a utilização de um termômetro, sendo considerado como tempo de indução o tempo no qual se observou um aumento brusco na temperatura.

Posteriormente, o material foi vazado em um molde na forma de pequenos cilindros cuja altura é duas vezes maior do que o diâmetro da base com as dimensões de 8 x 16 mm. Os cilindros foram colocados em um dessecador por $24 \mathrm{~h} \mathrm{e}$ finalmente secos em uma estufa a $100{ }^{\circ} \mathrm{C}$ por $24 \mathrm{~h}$. Depois de secos os corpos de prova foram sinterizados a $1300{ }^{\circ} \mathrm{C}$ com patamar de $1 \mathrm{~h}$ com rampa de aquecimento de $6{ }^{\circ} \mathrm{C} / \mathrm{min}$.

A porosidade total foi determinada pelo método geométrico e comparada com o valor da densidade teórica da HA $\left(3,156 \mathrm{~g} / \mathrm{cm}^{3}\right)$. Para cada medida de porosidade foram utilizados 6 corpos de prova. O tamanho de poros e a interconectividade entre os poros foram examinados por microscopia eletrônica de varredura (Jeol 6505). A distribuição de tamanho de poros foi medida para cada micrografia com o software analisador de imagens Image Tool.

\section{RESULTADOS E DISCUSSÃO}

Os teores de surfactante e sólidos foram 1\% e 62\%, respectivamente, conforme estudos prévios [8,9]. Depois de calcinada a $1200{ }^{\circ} \mathrm{C}$ a HA foi moída por $24 \mathrm{~h}$ em álcool para moagem e desaglomeração das partículas, resultando em uma distribuição estreita de tamanho de partículas com valor médio de 2,7 $\mu$ m. Não foi possível medir a área superficial da hidroxiapatita calcinada a $1200^{\circ} \mathrm{C}$ pela técnica BET por ter uma área superficial específica muito baixa, provavelmente menor do que $1,0 \mathrm{~m}^{2} / \mathrm{g}$. Tal valor é menor que o fundo de escala do equipamento de medida. A hidroxiapatita utilizada 
por Padilla et al. [12] foi obtida pelo método via-úmida sem a adição de defloculante e apresentou área superficial de $3,5 \mathrm{~m}^{2} / \mathrm{g}$ e tamanho médio de partículas $3 \mu \mathrm{m}$; o pó foi calcinado a $1200{ }^{\circ} \mathrm{C}$. Já Leliévre et al. [13] obtiveram a hidroxiapatita pela precipitação entre $\left(\mathrm{NH}_{4}\right) \mathrm{HPO}_{4}$ e $\mathrm{Ca}\left(\mathrm{NO}_{3}\right)_{2}$ e a área superficial e tamanho médio de partículas foram $0,68 \mathrm{~m}^{2} / \mathrm{g}$ e $2 \mu \mathrm{m}$, respectivamente, sendo calcinada a $1180{ }^{\circ} \mathrm{C}$.

A análise de difração de raios $\mathrm{X}$ mostra que não houve formação de outras fases, como $\beta$-TCP e $\mathrm{CaO}$ após a calcinação a $1200{ }^{\circ} \mathrm{C}$ e a sinterização a $1300{ }^{\circ} \mathrm{C}$. O difratograma corresponde à hidroxiapatita hexagonal. Os picos assinalados no difratograma da Fig. 1 correspondem ao arquivo JCPDS 09-0432 e estão indexados [1]. Não são encontradas as fichas de número 09-0169 e 37-1497 correspondentes às fases $\beta$-TCP e $\mathrm{CaO}$, respectivamente.

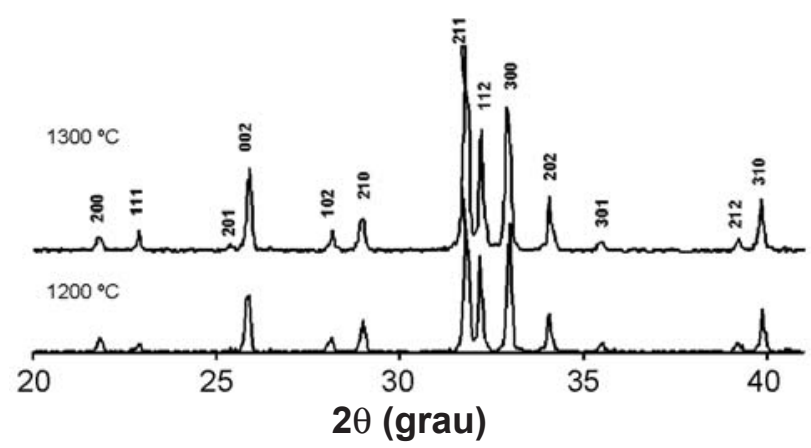

Figura 1: Padrões de difração de raios-X para o pó de hidroxiapatita após calcinação a 1200 e $1300^{\circ} \mathrm{C}$.

[Figure 1: X-ray diffraction patterns of hydroxyapatite powder after calcination at 1200 and $1300^{\circ} \mathrm{C}$.]

A análise por espectroscopia de infravermelho, Fig. 2, mostra os grupos químicos presentes no material sintetizado com a adição de defloculantes. $\mathrm{O}$ espectro da hidroxiapatita

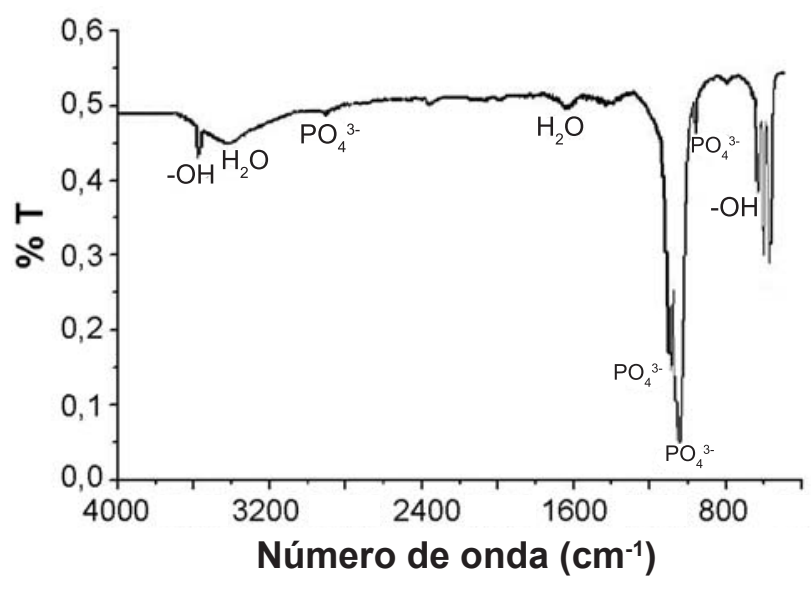

Figura 2: Espectroscopia de infravermelho do pó de hidroxiapatita calcinado a $1200^{\circ} \mathrm{C}$.

[Figure 2: Infrared spectroscopy of the HA powder calcined at $1200^{\circ} \mathrm{C}$.] apresenta bandas estreitas, mostrando alta cristalinidade [2].

A marca característica da HA é um pico bem definido em torno de $630 \mathrm{~cm}^{-1}$ [3]. Esse pico é um triplete que se relaciona com as ligações $\mathrm{PO}_{4}^{3-}$ em 601 e $570 \mathrm{~cm}^{-1}$ com contribuição da ligação -OH do grupo apatita em $630 \mathrm{~cm}^{-1}$. Outro pico que é correspondente ao grupo -OH estrutural é em $3570 \mathrm{~cm}^{-1}$. O pico que se estende de aproximadamente 3700 a 3000 $\mathrm{cm}^{-1} \mathrm{e}$ o pico em $1627 \mathrm{~cm}^{-1}$ correspondem à água absorvida. Os picos em $1047 \mathrm{~cm}^{-1}$ e em $1090 \mathrm{~cm}^{-1}$ correspondem à vibração de deformação do grupo $\mathrm{PO}_{4}^{3-}$, enquanto o em 962 $\mathrm{cm}^{-1}$ corresponde ao modo de estiramento do grupo $\mathrm{PO}_{4}^{3-}$ $[14,15]$. As bandas encontradas em 962 e $2970 \mathrm{~cm}^{-1}$ são características da hidroxiapatita deficiente em cálcio -CDHA $[2,16]$, também denominado fosfato de cálcio amorfo - ACP $\left(\mathrm{Ca}_{3}\left(\mathrm{PO}_{4}\right)_{2} \cdot \mathrm{nH}_{2} \mathrm{O}\right)$.

A ausência de $\beta$-TCP se confirma, pois de acordo com Le Geros [2], a única diferença entre os espectros da hidroxiapatita e do $\beta$-TCP está nas bandas de absorção do grupo P-O na faixa de 500 a $1200 \mathrm{~cm}^{-1}$. Nestas regiões, para a fase $\beta$-TCP, aparecem as bandas a 1115, 1096, 1007 e 944 $\mathrm{cm}^{-1}$; já para a hidroxiapatita, as bandas se localizam em $1090,1040,960,605$ e $505 \mathrm{~cm}^{-1}$.

A Tabela II apresenta os teores de iniciador e de acelerador utilizados em percentual em relação à massa de sólidos utilizada. A quantidade de iniciador possui maior influência no tempo de indução do que a quantidade de acelerador, pois a polimerização dos monômeros monofuncionais e difuncionais somente ocorre na presença de radicais livres. Os radicais livres são gerados pelo iniciador, enquanto o acelerador facilita a decomposição do iniciador, assim os radicais são produzidos em uma taxa suficiente em baixas temperaturas. Nos sistemas de polimerização via radicais livres é comum se observar um período de inatividade entre o momento de introdução do iniciador e do catalisador e o momento em que se inicia a reação. Esse tempo é conhecido como tempo de indução. Para a grande maioria dos sistemas, a viscosidade continua a mesma durante o tempo de indução, até que aumenta rapidamente com o início da formação do gel [17]. Outro fator que indica o início da formação do gel e o término do tempo de indução é o grande aumento da temperatura [17-19]. O tempo de indução pode ser

Tabela II - Quantidades de iniciador, acelerador, razão iniciador/acelerador e seus tempos de indução.

[Table 2 - Amounts of initiator, accelerator, initiator/ accelerator ratio and the induction time.]

\begin{tabular}{cccc}
\hline \% Iniciador & \% Acelerador & $\begin{array}{c}\text { Iniciador: } \\
\text { Acelerador }\end{array}$ & $\begin{array}{c}\text { Tempo de } \\
\text { Indução (s) }\end{array}$ \\
\hline 1 & 1 & $1: 1$ & 1680 \\
1,5 & 1,5 & $1: 1$ & 540 \\
1,5 & 2,5 & $3: 5$ & 300 \\
2,5 & 1,5 & $5: 3$ & 180 \\
2,5 & 2,5 & $1: 1$ & 90 \\
2,5 & 3,5 & $5: 7$ & 60 \\
3,5 & 2,5 & $7: 5$ & 30 \\
\hline
\end{tabular}


controlado principalmente pelas quantidades de iniciador e de acelerador de reação [19].

O sistema de polimerização acrilamida-MBAM apresenta menor tempo de indução do que os outros sistemas que vem sendo pesquisados para uso em gelcasting, como a metacrilamida [17] e o ácido acrílico [19]. Comparando duas amidas, a acrilamida $(\mathrm{AM}), \mathrm{CH}_{2}=\mathrm{C}(\mathrm{H}) \mathrm{CONH}_{2}$, com a metacrilamida (MAM), $\mathrm{CH}_{2}=\mathrm{C}\left(\mathrm{CH}_{3}\right) \mathrm{CONH}_{2}$, quanto às suas cinéticas de reação, o fator do impedimento estérico afeta fortemente a velocidade da reação, pois o átomo de carbono responsável pela polimerização está menos coberto do que na AM do que na MAM [17].

A Fig. 3 mostra a porosidade em função do tempo de indução. Quanto maior for o tempo de indução maior será a porosidade final. Para tempos de indução muito curtos a porosidade reduz-se consideravelmente. Isso prova que com o passar do tempo ocorre o processo de introdução de ar na suspensão, cujo efeito reflete não somente na porosidade, devido ao aumento no volume de ar dentro dos poros, mas também no tamanho de poros. De acordo com a Fig. 3 a porosidade cresce rapidamente até $90 \mathrm{~s}$ passando a ter um crescimento mais lento após esse tempo. Não foi possível medir a porosidade dos blocos obtidos com 540 e $1680 \mathrm{~s}$ pelo método geométrico, utilizado neste trabalho, pois eles ficaram disformes, ocasionado pelo peso próprio dos corpos de prova em função do longo tempo levado para a polimerização ocorrer.

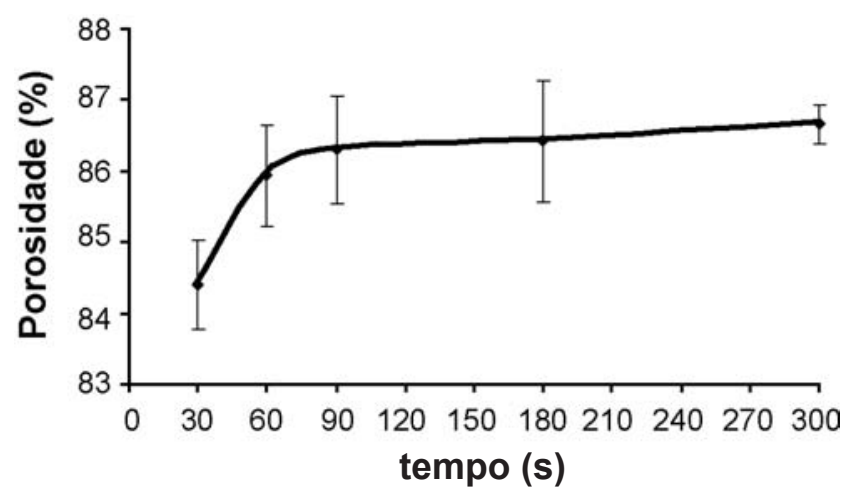

Figura 3: Porosidade em função do tempo de indução.

[Figure 3: Porosity as a function of induction time.]

Tabela 3 - Tamanho médio e o tamanho modal de poros e tempos de indução.

[Table 3 - Medium pore size and modal pore size for each induction time]

\begin{tabular}{lccccccc}
\hline Tempo $(\mathrm{s})$ & 30 & 60 & 90 & 180 & 300 & 540 & 1680 \\
\hline Tamanho médio $(\mu \mathrm{m})$ & 191 & 191 & 185 & 195 & 217 & 387 & 485 \\
Desvio padrão & 80 & 76 & 62 & 81 & 98 & 219 & 196 \\
Tamanho modal $(\mu \mathrm{m})$ & 160 & 168 & 141 & 132 & 112 & 194 & 235 \\
& & & 216 & 182 & 182 & 284 & 352 \\
& & & & 255 & 112 & 182 & 480 \\
\hline
\end{tabular}

A Tabela III mostra o tamanho médio de poros para os tempos de indução obtidos.

A Fig. 4 mostra a micrografia obtida por microscopia eletrônica de varredura para cada tempo de indução obtido e ao lado a respectiva distribuição de tamanho de poros obtida pela análise das micrografias com o software analisador de
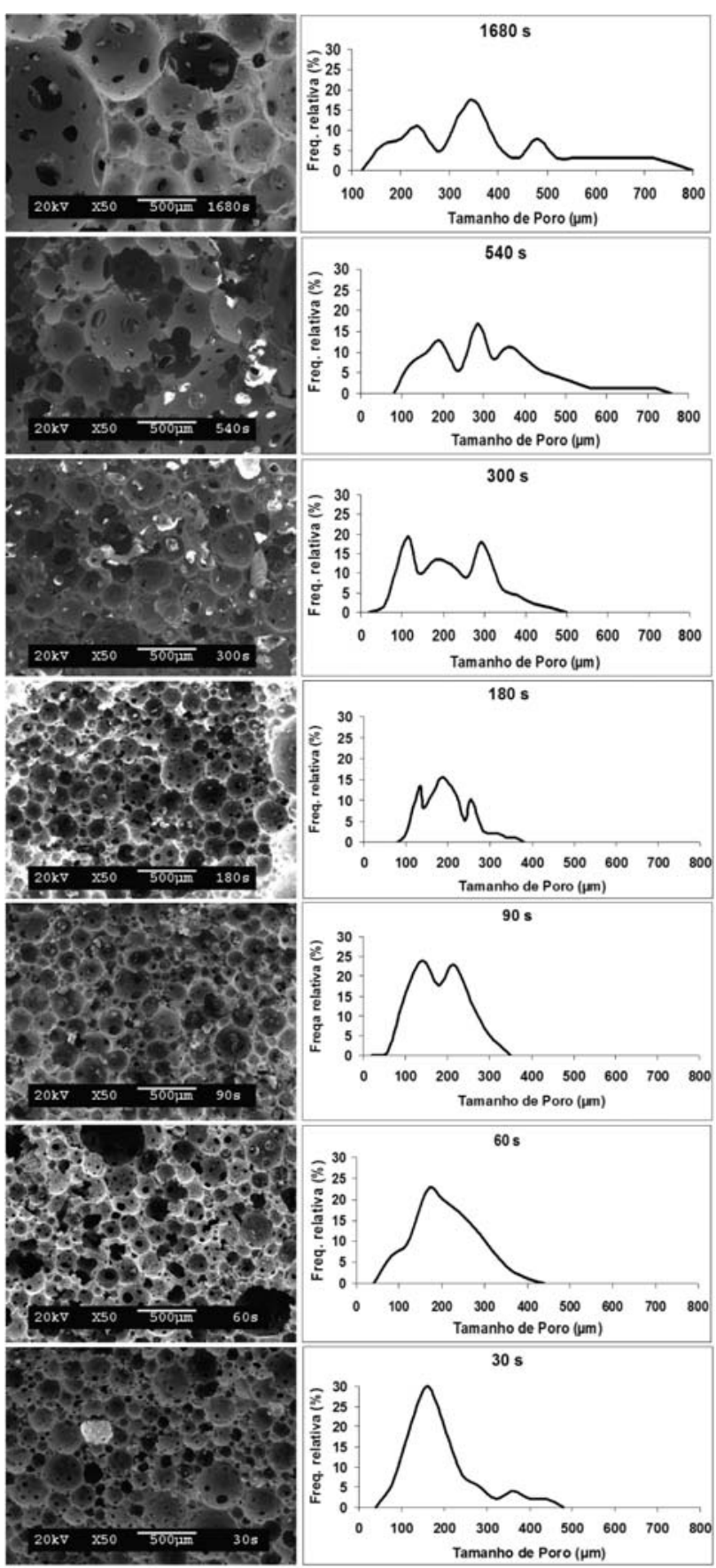

Figura 4: Micrografias obtidas por microscopia eletrônica de varredura do corpo poroso para cada tempo de indução e sua respectiva distribuição de tamanho de poros.

[Figure 4: SEM micrographs of the porous HA and pore size distribution graphs for each induction time.] 
imagens, sendo medidos no mínimo 100 poros para cada amostra.

O início do processo de coalescimento se dá apenas após $90 \mathrm{~s}$; a partir deste tempo começa a ocorrer uma distribuição de tamanho de poros (DTP) bimodal, porém o valor médio de tamanho de poros se mantém em torno de $190 \mu \mathrm{m}$. A partir de 3 min o comportamento de DTP passa a ser trimodal, de maneira que o tamanho médio de poros também começa a aumentar chegando a $215 \mu \mathrm{m}$. O tamanho médio de poros aumenta até um máximo de $485 \mu \mathrm{m}$ para o tempo de $1680 \mathrm{~s}$, mantendo o comportamento trimodal da DTP.

A Figura 4 mostra o crescimento do tamanho médio de poros e os comportamentos da DTP dados da Tabela III. Pode-se ressaltar que todos os blocos apresentaram alto grau de interconexões, sendo que quanto maior o tempo de indução, se tornam mais pronunciadas.

Os resultados das constantes de permeabilidade medidos para as amostras com tempo de indução de 30, 60, 90, 180 e $300 \mathrm{~s}$ são mostrados na Tabela IV. Não foi possível medir a permeabilidade das amostras com tempo de indução de 540 e 1680 s por causa da baixa resistência mecânica.

Tabela 4 - Constantes darcyana $\left(\mathrm{k}_{1}\right)$ e não-darcyana $\left(\mathrm{k}_{2}\right)$ para os tempos de indução medidos.

[Table 4 - Darcyan constant $\left(k_{p}\right)$ and non-Darcian constant (k2) for for each induction time.]

\begin{tabular}{llllll}
\hline $\begin{array}{l}\text { Tempo de } \\
\text { polimerização (s) }\end{array}$ & 30 & 60 & 90 & 180 & 300 \\
\hline $\mathrm{k}_{1}$ & $9,94 \mathrm{E}^{-12}$ & $2,08 \mathrm{E}^{-11}$ & $2,40 \mathrm{E}^{-11}$ & $2,31 \mathrm{E}^{-11}$ & $1,82 \mathrm{E}^{-11}$ \\
$\mathrm{k}_{2}$ & $9,32 \mathrm{E}^{-06}$ & $1,39 \mathrm{E}^{-05}$ & $3,98 \mathrm{E}^{-05}$ & $3,01 \mathrm{E}^{-05}$ & $2,06 \mathrm{E}^{-05}$ \\
\hline
\end{tabular}

Verifica-se que há a tendência da diminuição das constantes de permeabilidade darcyana e não-darcyana com o aumento do teor de sólidos. Tal comportamento pode ser relacionado com a porosidade do material, pois quanto mais poroso ele for, maior será o seu grau de interconexão entre os poros, o que resultará em uma maior porosidade, refletindo em valores altos de $\mathrm{k}_{1}$ e $\mathrm{k}_{2}$.

As Figs. 5 e 6 mostram, respectivamente, uma comparação entre os valores encontrados para $\mathrm{k}_{1}$ e $\mathrm{k}_{2}$ com os valores de alguns trabalhos publicados. Ambas publicações de Ortega et al. [20, 21] utilizaram alumina, já a de Sepúlveda et al. [22] utilizou hidroxiapatita. Os valores de $\mathrm{k}_{1}$ obtidos neste trabalho são menores que os encontrados por Ortega et al. [21] para valores semelhantes de porosidade. Já os valores de $\mathrm{k}_{2}$ são um pouco maiores, porém a diferença é muito pequena. Podese dizer que o significado dessa pequena diferença é que a hidroxiapatita porosa obtida neste trabalho tem maior influência dos efeitos viscosos (viscosidade do fluido e área de contato HA-fluido), já a alumina porosa obtida por Ortega et al. [21] possui maior sensibilidade aos efeitos cinéticos (densidade do fluido, turbulência e tortuosidade). $\mathrm{O}$ cálculo das constantes $\mathrm{k}_{1}$ e $\mathrm{k}_{2}$ se deu por meio de regressão linear, no qual o coeficiente $\mathrm{r}^{2}$ era no mínimo igual a 0,99 , o que significa que o erro das medidas é muito pequeno. O mesmo é pressuposto para os valores obtidos nos dois trabalhos citados [21, 22].

A Fig. 7 mostra a relação entre o tamanho médio de poros e as constantes darcyanas e não-darcyanas de permeabilidade.

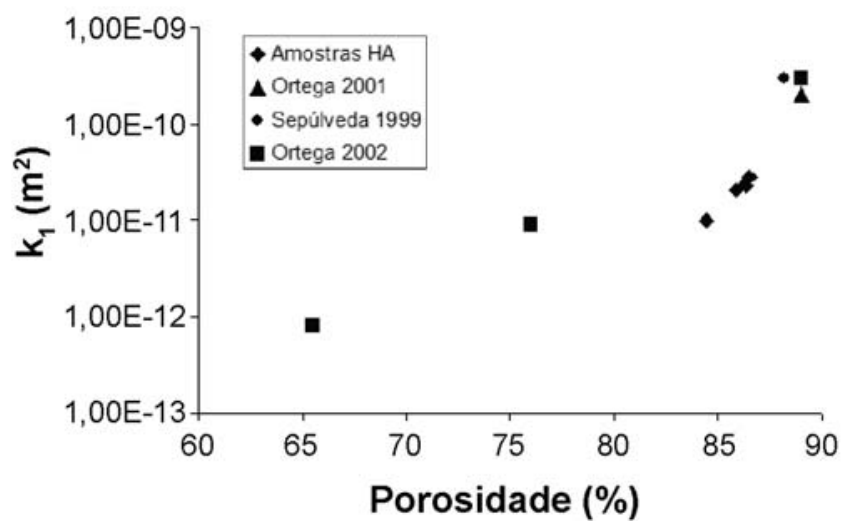

Figura 5: Comparação entre os resultados obtidos de $\mathrm{k}_{1}$ neste trabalho com os trabalhos pubçicados.

[Figure 5: Comparison between the results obtained of $k_{1}$ in this work with published ones.]

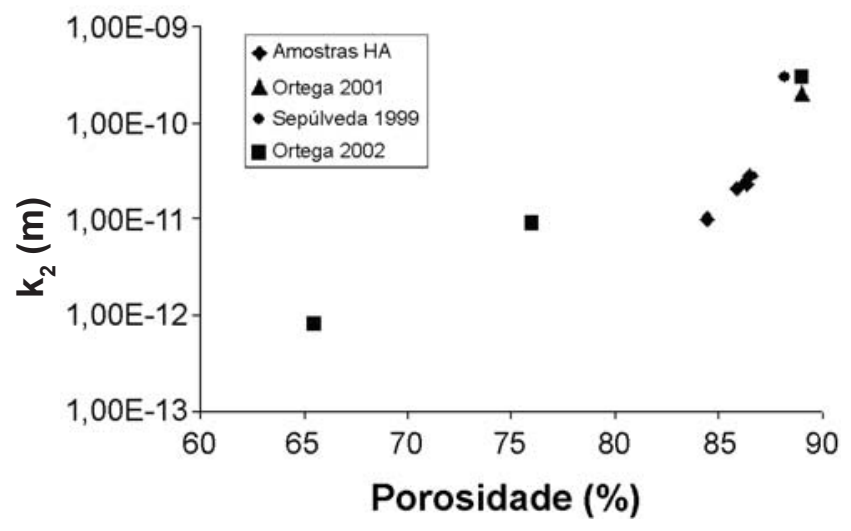

Figura 6: Comparação entre os resultados obtidos de $\mathrm{k}_{2}$ neste trabalho com os os trabalhos pubçicados.

[Figure 6: Comparison between the results obtained of $k_{2}$ in this work with published ones.]

A permeabilidade também é função do tamanho médio de poros, pois quanto maior o poro, maior a constante de permeabilidade darcyana ou não-darcyana, visto que ambas aumentam.

Um fator importante para a polimerização no método gelcasting é a atmosfera utilizada. São encontrados estudos que mostram que a presença de $\mathrm{O}_{2}$ inibe a reação de polimerização [11], porém esse trabalho mostra que adições um pouco maiores de iniciador e acelerador permitem a ocorrência da reação de polimerização, e a obtenção de corpos porosos com propriedades finais adequadas para serem utilizados como implante, sendo necessários estudos in vitro e in vivo para confirmar. 
Este estudo mostra que o tempo de indução é de fundamental importância nas propriedades finais, causando mudanças significativas principalmente no tamanho médio de poros e na microestrutura do corpo final, sendo um parâmetro essencial para a reprodutibilidade dos resultados.

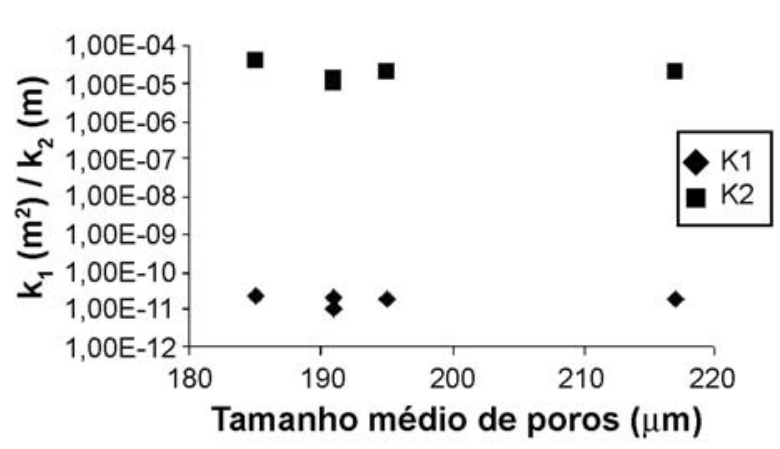

Figura 7: Constantes de permeabilidade $k_{1}$ e $k_{2}$ em função do tamanho médio de poros.

[Figure 7: Permeability constants $k_{1}$ and $k_{2}$ as a function of medium pore size.]

\section{CONCLUSÕES}

Foram obtidos blocos porosos de hidroxiapatita pelo método gelcasting de espumas com tamanho de poros controlado e com potencial para uso em medicina e odontologia como próteses reparadoras de defeitos ósseos, em locais nos quais a solicitação mecânica seja baixa. A variação das quantidades de iniciador e de acelerador permitiu que fossem obtidos diferentes tempos de indução, sendo que é possível controlar o tempo de indução através de diferentes adições de iniciador e acelerador. O tempo de indução é fortemente influenciado pelas quantidades de catalisador e iniciador, sendo mais influenciada pela quantidade de iniciador, tal parâmetro também grande influencia na porosidade e no tamanho médio de poros, tempos de indução maiores do que 5 min geram blocos porosos com tamanho de poro médio superior a $300 \mu \mathrm{m}$, porém o aumento se dá à custa de uma alta retração, sendo assim difícil utilizar tal tempo para a confecção de peças com formatos complexos. É possível realizar a polimerização utilizando atmosfera de reação contendo $\mathrm{O}_{2}$.

\section{REFERÊNCIAS}

[1] H. Aoki, Science and medical applications of hydroxyapatite, $1^{\text {st }}$ Ed., JAAS, Tokyo (1991) 11.

[2] R. Z. Le Geros, Calcium Phosphates in Oral Biology and Medicine, Monographs in Oral Science, Vol. 15, S. Karger, Switzerland (1991) 26.

[3] L. L. Hench, J. Wilson, An Introduction to Bioceramics,
World Sci. Publ. Co., Singapore (1993) 54.

[4] V. Karageorgiu, D. Kaplan, Porosity of 3D biomaterial scaffolds and osteogenesis, Biomaterials 26 (2005) 54745491

[5] P. Sepúlveda, Gelcasting Foams for Porous Ceramics. Am. Ceram. Soc. Bull. 76 (1997) 61.

[6] A. C. Young, O. O. Omatete, M. A. Janney, P. A. Menchhofer, Gelcasting of Alumina, J. Am. Ceram. Soc. 74, 3 (1991) 3349-3352.

[7] M. A. Ishida, T. M. Volkmer, L. A. Santos, Modificação da condição de precipitação de hidroxiapatita sintética, Anais do III Cong. Lat. Am. Biomateriais e Órgãos Artificiais, Campinas, SP (2004) 1-12.

[8] T. M. Volkmer, L. A. Santos, D. Breuer, Influência do teor de surfactante na porosidade de hidroxiapatita obtida pelo método gelcasting de espumas, Anais do $50^{\circ}$ Cong. Bras. Cerâm., Blumenau, SC (2006) 1-10.

[9] T. M. Volkmer, L. A. Santos, Avaliação da microestrutura de blocos porosos obtidos por gelcasting de espumas, Anais do IV Cong. Lat. Am. de Biomateriais e Órgãos Artificiais, Caxambu, MG (2006) 1 - 12.

[10] T. M. Volkmer, M. B. Borsa, D. Breuer, L. A. Santos, Estudo dos parâmetros de reação para obtenção de blocos porosos de alumina e hidroxiapatita pelo método gelcasting de espumas, Anais do XVI Cong. Bras. Eng. Ci. Mater., Porto Alegre, RS (2004) 1-8.

[11] J. S. Ha, Effect of atmosphere type on gelcasting behavior of $\mathrm{Al}_{2} \mathrm{O}_{3}$ and evaluation of green strength, Ceram. Int. 26 (2000) 251-254.

[12] S. Padilla, M. Vallet-Regí, M. P. Ginebra, F. J. Gil, Processing and mechanical properties of hydroxyapatite pieces obtained by the gelcasting method, J. Eur. Ceram. Soc. 25 (2005) 375-383.

[13] F. Lelièvre, D. Bernache-Assolant, T. Chartier, Influence of powder characteristics on the reological behavior of hydroxyapatite slurries, J. Mater. Sci.: Mater. Medicine 7 (1996) 489-494.

[14] H. R. Ramay, M. Zhang, Preparation of porous hidroxyapatite scaffolds by combination of the gelcasting and polymer sponge methods, Biomaterials 24 (2003) 32933302 .

[15] J. Zhang, M. Macda, N. Kotobuki, M. Hirose, H. Ohgushi, D. Jiang, M. Iwasa, Mater. Chem. Phys. 99 (2006) 398-404.

[16] M. Potoczek, E. Zawadzak, Initiator effect on the gelcasting properties of alumina in a system involving lowtoxic monomers, Ceram. Int. 30 (2004) 793-799.

[17] P. A. Sepúlveda, J. G. P. Binner, Processing of cellular ceramics by foaming and in situ polymerization of organic monomers, J. Eur. Ceram. Soc. 19 (1999) 2059-2066.

[18] M. Kokabi, A. A. Babaluo, A. Barati, Gelation process in low-toxic gelcasting systems, J. Eur. Ceram. Soc. 26 (2006) 3083-3090.

[19] F. S. Ortega, P. Sepúlveda, V. C. Pandolfelli, Reologia, cinética de polimerização e resistência mecânica de sistemas para a produção de espumas cerâmicas via "gelcasting", Cerâmica 47, 302 (2001) 94-99. 
[20] F. S. Ortega, M. D. M. Innocentini, F. A. O. Valenzuela, V. C. Pandolfelli, Efeito da técnica de aeração sobre a macroestrutura e a permeabilidade espumas cerâmicas produzidas via "gelcasting", Cerâmica 48, 306 (2002) 79-85.
[21] P. Sepúlveda, V. C. Pandolfelli, S. O. Rogero, O. Z. Higa, J. C. Bressiani, Hidroxiapatita porosa produzida através do gelcasting de espumas visando aplicações biomédicas, Cerâmica 45, 296 (1999) 198-202.

(Rec. 26/03/2007, Ac. 10/08/2007) 